\title{
Heparin-induced thrombocytopenia in patients readmitted after open cardiac surgical procedures:
}

\section{A case series}

\author{
Michael A. Catalano, BS, ${ }^{a}$ Vikram Prasad, MD, ${ }^{\text {a }}$ Alexander M. Spring, BS, ${ }^{\text {a Hugh Cassiere, MD, }}{ }^{a}$ \\ Tylis Y. Chang, MD, ${ }^{b}$ Alan Hartman, MD, ${ }^{a}$ and Pey-Jen Yu, MD ${ }^{a}$
}

\section{ABSTRACT}

Objectives: Heparin-induced thrombocytopenia (HIT) is an immune-mediated complication that occurs in a small percentage of patients exposed to heparin. Concerns of HIT are particularly high in patients undergoing cardiac procedures requiring cardiopulmonary bypass, as they are exposed to high doses of heparin intraoperatively. Our aim was to identify and assess the hospital courses of patients who were diagnosed with HIT during readmission following cardiac surgery.

Methods: A retrospective review of patients who underwent open cardiac surgical procedures from June 2017 through October 2019 was performed. Of these, we identified patients who were newly diagnosed with HIT upon readmission. HIT positivity was defined as a positive anti-PF4 antibody screening test, plus a positive serotonin release assay.

Results: Of the 2496 patients identified, 13 patients were HIT positive on index admission and were excluded. Of the remaining 2483 patients, 351 were readmitted within 30 days. Six were newly diagnosed with HIT during readmission, 5 of whom presented with thrombotic complications. One patient was readmitted with thrombocytopenia and was started on argatroban; the remaining 5 did not have a significantly lower platelet count on readmission. Of the 12 patients readmitted for venous thromboembolism, 4 tested positive for HIT.

Conclusions: HIT can have a delayed appearance following open heart surgery. Venous thromboembolism appears to be a significant indicator for HIT during readmission, even in the absence of thrombocytopenia. This may support the use of non-heparin anticoagulation for cardiac surgery patients readmitted with thromboembolism until HIT status is determined. (JTCVS Open 2020;4:36-42)

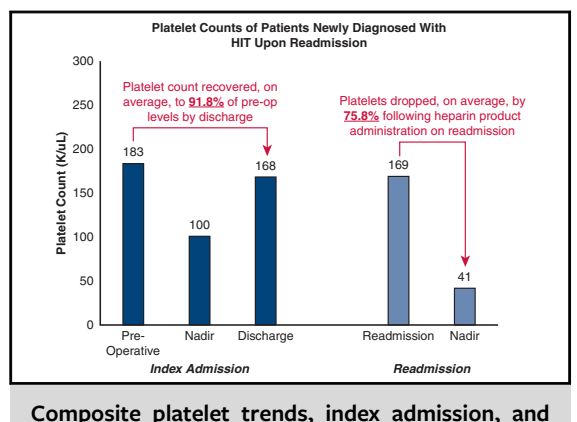

readmission.

CENTRAL MESSAGE

Patients readmitted with throm-

bosis after cardiac surgery are at

risk for HIT, despite normal

platelet counts; non-heparin an-

ticoagulation should be used

until HIT status is determined.

\section{PERSPECTIVE}

Risk of HIT is high in patients undergoing procedures requiring cardiopulmonary bypass; however, little is known about the presentation of HIT upon readmission. We describe a series of cardiac surgical patients readmitted with thrombosis who received heparin and experienced a significant platelet drop secondary to HIT, highlighting the importance of use of non-heparin products in such patients.

See Commentaries on pages 43 and 45 .
Heparin-induced thrombocytopenia (HIT) is an acquired and potentially life-threatening thrombotic complication

From the ${ }^{\mathrm{a}}$ Division of Cardiovascular and Thoracic Surgery and ${ }^{\mathrm{b}}$ Department of Pathology, Zucker School of Medicine at Hofstra/Northwell, Manhasset, NY.

Received for publication July 29, 2020; revisions received July 29, 2020; accepted for publication July 29, 2020; available ahead of print Sept 4, 2020.

Address for reprints: Pey-Jen Yu, MD, 300 Community Dr, Manhasset, NY 11030 (E-mail: pyu2@northwell.edu).

2666-2736

Copyright $(2020$ The Authors. Published by Elsevier Inc. on behalf of The American Association for Thoracic Surgery. This is an open access article under the CC BY-NCND license (http://creativecommons.org/licenses/by-nc-nd/4.0/).

https://doi.org/10.1016/j.xjon.2020.07.009 that occurs in a small percentage of patients exposed to unfractionated heparin (UFH) and, less commonly, lowmolecular weight heparin. It is an immune-mediated disorder that results in the production of antibodies against heparin-platelet factor 4 complexes, leading to a sharp decline in platelet count by at least $50 \%$ and a paradoxical prothrombotic state. If left untreated, HIT can lead to heparin-induced thrombocytopenia and thrombosis (HITT), resulting in significant morbidity and mortality.

Concerns of HIT are particularly high in patients undergoing cardiac surgical procedures requiring cardiopulmonary bypass (CPB), as they are exposed to high doses of 


\section{Abbreviations and Acronyms \\ $\mathrm{CPB}=$ cardiopulmonary bypass \\ DVT $=$ deep-vein thrombosis \\ ELISA $=$ enzyme-linked immunosorbent assay \\ HIT = heparin-induced thrombocytopenia \\ HITT = heparin-induced thrombocytopenia and thrombosis \\ $\mathrm{PE} \quad=$ pulmonary embolus \\ PF4 = platelet factor 4 \\ UFH $=$ unfractionated heparin \\ VTE $=$ venous thromboembolism}

intraoperative UFH. HIT rates have been shown to be approximately $3 \%$ in this patient population on index admission, and HIT is associated with a $50 \%$ increase in mortality and a doubling of the risk of stroke in this patient population. ${ }^{1,2}$ While the risk of HIT on index admission after cardiac surgery is well described, data on the incidence and presentation of HIT in patients readmitted after open heart surgery are limited. The aim of this study was to determine the incidence, clinical course, and outcomes of patients who were newly diagnosed with HIT during readmission following cardiac surgery.

\section{METHODS}

This study was conducted with the approval of the Northwell Health System institutional review board. As this is a retrospective study using deidentified data collected for the New York State and Society of Thoracic Surgeons databases, specific waiver of the need for individual patient consent was granted by the institutional review board.

A retrospective review of all patients who underwent open cardiac surgical procedures requiring CPB from June 2017 through October 2019 was performed. All patients received heparin during $\mathrm{CPB}$; the heparin used was supplied by a single vendor. Patients who underwent implantation of mechanical assist devices or cardiac transplants were excluded. Patients who were diagnosed with HIT on index admission were excluded. Of the remaining patients, we identified all patients who were readmitted within 30 days of discharge.

Patients who were newly diagnosed with HIT upon readmission were identified. Patients were screened for HIT with anti-PF4 antibody enzymelinked immunosorbent assay (ELISA) testing during their index admission if their postoperative platelet count continued to decline on postoperative day 3 to less than $50 \%$ of preoperative levels. Readmitted patients were screened for HIT with anti-PF4 antibodies ELISA testing if they were thrombocytopenic on admission (defined as platelet count $<100 \mathrm{~K} / \mu \mathrm{L}$ or $<50 \%$ of discharge platelet count), or they developed a greater than $50 \%$ drop in platelets during their readmission. HIT positivity was defined as a positive screening test for anti-PF4 antibodies with a positive serotonin release assay as confirmation. Electronic medical record data were reviewed to identify patients, as well as characteristics and data of interest. Data collected for each patient included demographic information (age and sex), cardiac surgical procedure type, daily platelet counts throughout index admission and readmission, anticoagulation use, readmission diagnosis, and HIT workup.

\section{RESULTS}

Of the 2496 patients who underwent open cardiac surgery requiring $\mathrm{CPB}$ during the study period, $13(0.5 \%)$ patients in the sample were found to be HIT positive on index admission and were excluded from the study. Of the remaining patients, 351 patients $(14.1 \%)$ were readmitted within 30 days of discharge from the index admission. Of the readmitted patients, $12(3.4 \%)$ presented with thrombotic complications of deep vein thrombosis (DVT) or pulmonary embolus (PE). Six patients $(1.7 \%)$ tested positive for HIT antibodies upon readmission, 5 of whom had HITT as they were admitted with thrombotic complications (Figure 1). Demographic information, surgical procedure performed, readmission diagnosis, platelet trends, anticoagulation selection, and HIT workup for the 6 patients are presented in Table 1. Figure 2 depicts platelet trends, anticoagulation use, readmission date, and HIT testing for each patient.

Of the 6 patients who were newly diagnosed with HIT or HITT during readmission, $5(83.3 \%)$ were male. Age ranged from 42 to 89 years, and mean age was found to be $69.5 \pm 15.4$ years. Cardiac surgical procedures performed included combined mitral valve replacement and aortic valve replacement, isolated aortic valve replacement, and coronary artery bypass graft. All patients received heparin loading intraoperatively during CPB. In addition, 5 of the 6 patients received either subcutaneous heparin or enoxaparin for venous thromboembolism (VTE) prophylaxis during their postoperative management. Patient 4 did not receive pharmacologic VTE prophylaxis and therefore did not have postoperative heparin exposure, given her baseline preoperative thrombocytopenia that persisted postoperatively.

The mean preoperative platelet count was $183.0 \pm$ $58.4 \mathrm{~K} / \mu \mathrm{L}$, the mean nadir platelet count during index admission was $100.3 \pm 39.2 \mathrm{~K} / \mu \mathrm{L}$, and the mean platelet count upon initial discharge was $167.8 \pm 61.4 \mathrm{~K} / \mu \mathrm{L}$. Nadir, on average, occurred $2.0 \pm 1.1$ days into admission; mean length of hospital stay was $5.0 \pm 0.9$ days. Platelet count recovered, on average, to $91.8 \%$ of preoperative levels upon discharge. One patient (Patient 1) met indications for HIT testing during index admission, was found to be HIT-negative on postoperative day 3 , and was discharged on postoperative day 6 . The other 5 patients did not meet criteria for HIT testing, and each of them experienced an increase in platelet count before discharge.

Of the 6 patients who were positive for HIT during readmission, $4(66.7 \%)$ presented with either DVT or PE (patients $3,4,5,6$ ). These 4 patients made up $33.3 \%$ of all cardiac surgical patients readmitted with DVT or PE. One of the remaining 2 patients diagnosed with HIT was found to have mesenteric ischemia and bowel perforation, secondary to thrombosis (patient 2). The final patient was readmitted with a pericardial effusion and was found to have significant thrombocytopenia upon admission (patient 1). Five of the 6 patients had readmission platelet counts that were not significantly different from their preoperative platelet count. The mean platelet count among these 5 


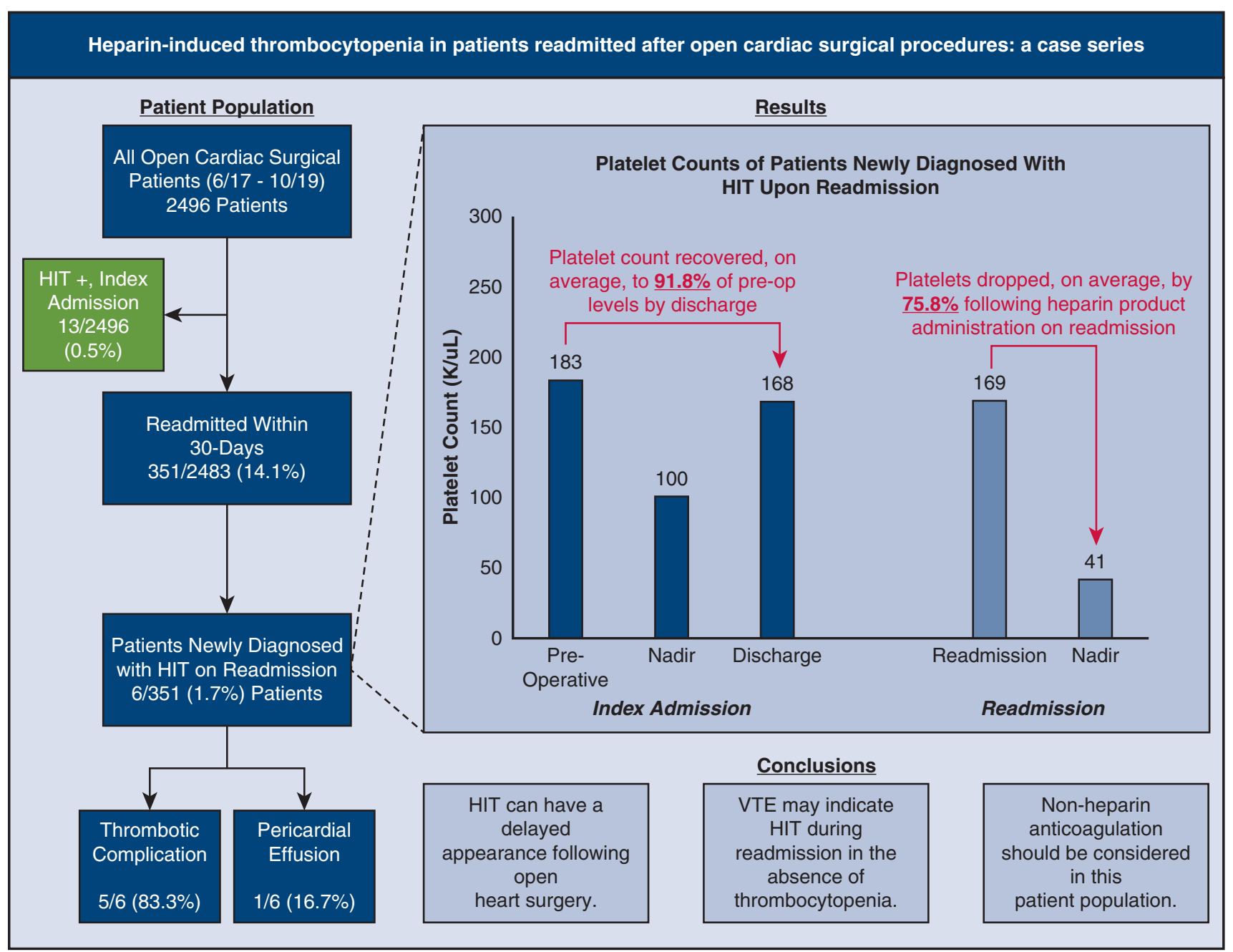

FIGURE 1. Overview of patient selection, platelet count trends, and conclusions of our analysis of patients diagnosed with heparin-induced thrombocytopenia and thrombosis during readmission. The mean preoperative platelet count was $183.0 \pm 58.4 \mathrm{~K} / \mu \mathrm{L}$, which recovered, on average, to $91.8 \%$ of preoperative levels by discharge. These patients presented upon readmission with a mean platelet count of $169 \mathrm{~K} / \mu \mathrm{L}$, not significantly lower than their discharge platelet count. However, following heparin product administration, there was a rapid and severe reduction in platelet count to a mean of $41 \mathrm{~K} / \mu \mathrm{L}$. HIT, Heparin-induced thrombocytopenia.

patients was $199.2 \pm 73.9 \mathrm{~K} / \mu \mathrm{L}$ (range of $112-285 \mathrm{~K} / \mu \mathrm{L}$ ), compared with their mean discharge platelet count of $182.0 \pm 56.6 \mathrm{~K} / \mu \mathrm{L}$ (range of $113-268 \mathrm{~K} / \mu \mathrm{L}$ ). All 5 patients were started on heparin-based anticoagulation for confirmed thrombosis. Each patient experienced a significant drop in platelets in the days following admission with a mean nadir of $47.2 \pm 19.0 \mathrm{~K} / \mu \mathrm{L}$ (range of 30 $77 \mathrm{~K} / \mu \mathrm{L}$ ). HIT assays were sent between day 2 and day 6 of rehospitalization for each of these patients, and all were found to be positive.

Patient 1 , in contrast, was readmitted with a pericardial effusion. Platelet count upon readmission was $17 \mathrm{~K} / \mu \mathrm{L}$ (dropping to $11 \mathrm{~K} / \mu \mathrm{L}$ later that day), compared with discharge platelet count of $97 \mathrm{~K} / \mu \mathrm{L} 3$ days previously. Because HIT was suspected initially, this patient was initiated on argatroban, and HIT assays were found to be positive by readmission day 2 . This patient experienced a steady increase in platelet count throughout admission, being discharged with a platelet count of $>100 \mathrm{~K} / \mu \mathrm{L}$.

There was no mortality or significant morbidity identified in the case series. However, each patient experienced a prolonged readmission course, with an average length of stay of $20.3 \pm 9.4$ days.

\section{COMMENT}

HIT is a potentially life-threatening adverse drug reaction that occurs in a small subset of patients receiving UFH and, less commonly, low-molecular weight heparin. Despite the development of new anticoagulants, UFH with its advantageous pharmacokinetics and ease of monitoring remains the 


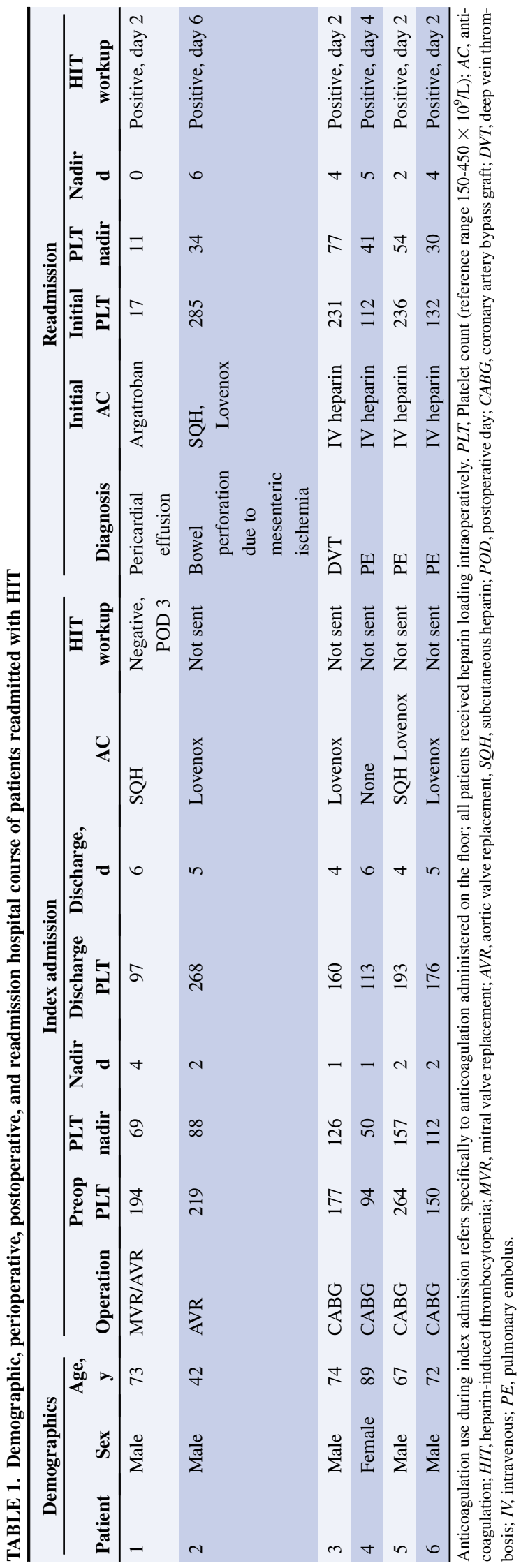

gold standard for intraoperative and perioperative anticoagulation in cardiac surgery. Patients undergoing cardiac surgical procedures requiring CPB are particularly vulnerable to HIT, as they are exposed to high doses of intraoperative UFH. The incidence of HIT in this patient population is reported to be approximately $3 \%$, and it is associated with a $50 \%$ increase in mortality and a doubling of the risk of stroke. ${ }^{1,2}$ Considering that thromboembolic complications are estimated to be as high as $6.1 \%$ per day in patients with undiagnosed HIT, clinicians managing these patients must have a detailed understanding of the disorder to ensure its early recognition, diagnosis, and treatment. ${ }^{3}$ Although the incidence and adverse outcomes associated with HIT in patients undergoing cardiac surgery on index admission have been well documented, follow-up data on the incidence of HIT on readmission are scarce. Our study is the first to help characterize the incidence and presentation of patients who were diagnosed with HIT upon readmission after cardiac surgery.

Currently, the 2 classes of tests used to assist in the diagnosis of HIT are immunologic and functional assays. Immunoassays, such as ELISA, are widely available and offer a high degree of sensitivity ( $>99 \%$ ) but are associated with limited specificity $(40 \%-80 \%){ }^{4}$ Such tests are an effective screening tool to exclude a diagnosis of HIT; however, the high false-positive rate limits the diagnostic ability. In fact, up to $50 \%$ of patients develop anti-PF4-heparin antibodies after CPB due to the high doses of UFH used intraoperatively. ${ }^{5}$ Functional assays, such as the serotonin release assay, in contrast, only detect the heparin antibodies that can activate platelets and are therefore considered the gold standard with a sensitivity and specificity greater than $95 \%{ }^{6}$ In this study, we only considered patients HIT positive if they were positive on both HIT ELISA and serotonin release assay assays.

The initial and most common manifestation of HIT is thrombocytopenia. After heparin exposure, platelet numbers decline rapidly, often by $50 \%$ or more from baseline. Platelet counts fall below $150 \times 10^{9} / \mathrm{L}$ in $90 \%$ of patients with a median nadir of $55 \times 10^{9} / \mathrm{L}$. $^{7}$ Diagnosing HIT in patients undergoing cardiac surgery can be particularly challenging due to the high incidence of postoperative thrombocytopenia. Generally, a $40 \%$ to $50 \%$ decrease in platelet count is expected in the immediate postoperative period following CPB due to platelet consumption and hemodilution with platelets typically recovering to preoperative levels within 5 to 6 days. ${ }^{8,9}$ A second decline in platelet count between days 5 and 10 is highly suspicious for HIT. ${ }^{8,10}$ As patients after cardiac surgery may be discharged home before the second decline in platelet count, clinical vigilance for HIT is vital in cardiac surgery patients that are readmitted after their surgery, as they will likely be re-exposed to heparin during their readmission. For example, each of the patients in our series was discharged 

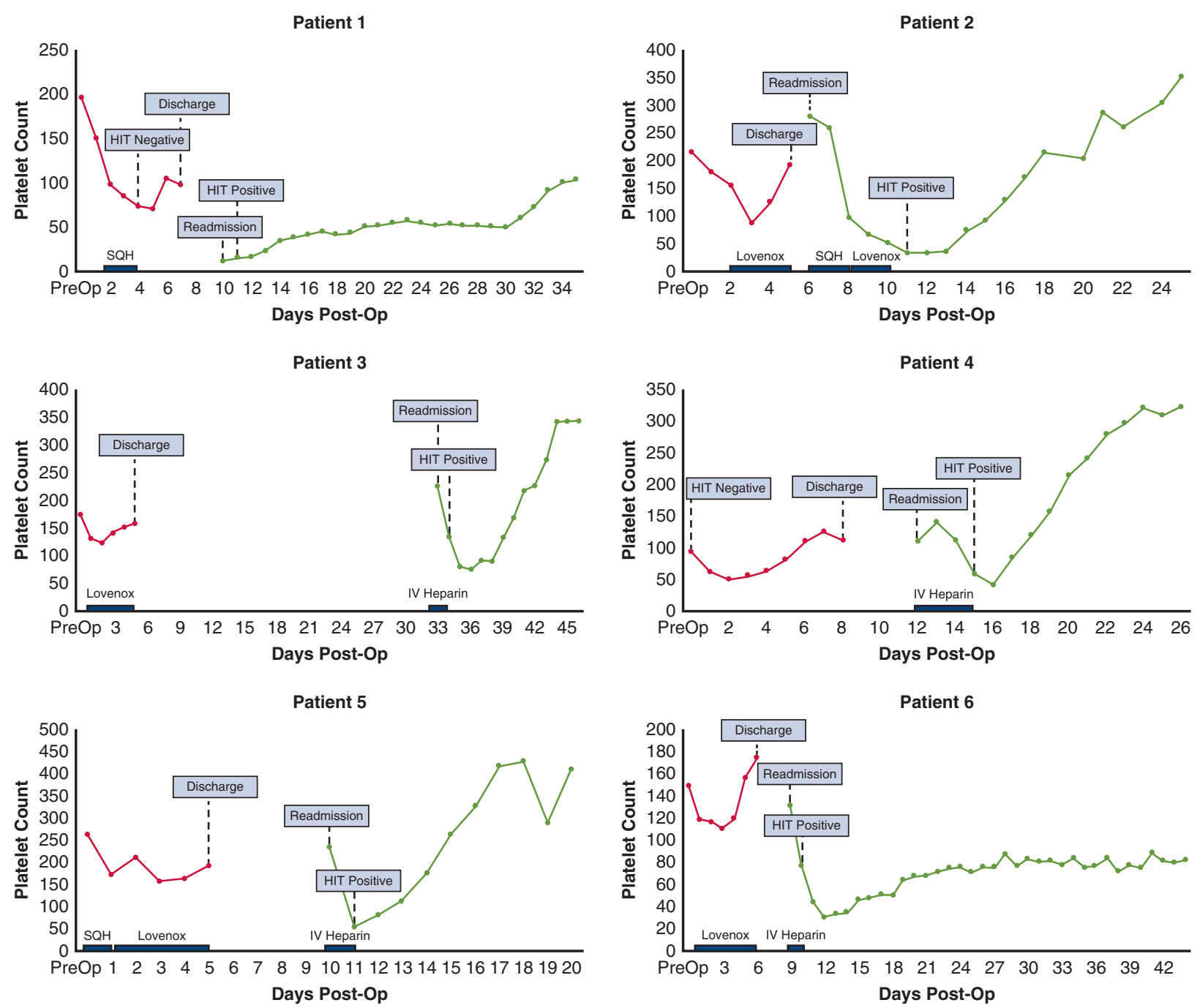

FIGURE 2. Depiction of index hospital admission and readmission course, anticoagulation administration, and trending platelet counts of 6 cardiac surgical patients readmitted with HIT. The readmission platelet trends highlight that patients 2-6 presented on readmission with thrombosis without significant thrombocytopenia; the subsequent anticoagulation with heparin products led to rapid reductions in platelet counts and HIT diagnosis. HIT, Heparin-induced thrombocytopenia; $S Q H$, subcutaneous heparin; $I V$, intravenous.

between 4 and 6 days postoperatively. Thus, it is possible that the aforementioned second decline in platelet count was missed while the patient was outside of the hospital. Interestingly, however, we found only 1 of 6 patients that were HIT positive during readmission had a significant decline in initial platelet count on readmission. This suggests that initial platelet count on readmission may not be a sensitive predictor of HIT positivity.

The presence of heparin-induced thrombosis in the absence of thrombocytopenia has been described in limited case reports. Tibayan and colleagues ${ }^{11}$ describe the case of a 55-year-old man who developed PE on day 12 postoperatively following cardiac surgical intervention. Despite normal platelet counts, this patient tested positive for both heparin-induced antibody and heparin-induced platelet aggregation. Bigdeli and colleagues ${ }^{12}$ also report a similar case of a 57-year-old cardiac surgical patient who developed PE and was diagnosed with heparin-induced thrombosis without thrombocytopenia; in this case, the lack of platelet decline expected in HIT or HITT was attributed to the counteractive effect of postoperative reactive thrombocytosis. Busche and colleagues ${ }^{13}$ describe a patient with toxic epidermal necrolysis who was found have positive HIT testing in the absence of thrombocytopenia. Interestingly, none of the patients who were readmitted with HITT in this case series had thrombocytopenia on initial presentation. This may suggest that the risk of heparin-induced thrombosis without thrombocytopenia may be greater in patients after cardiac surgery, highlighting the need for increased suspicion for heparininduced thrombosis without thrombocytopenia in these patients. 
Clinical manifestations of HIT are observed in less than $3 \%$ of patients undergoing cardiopulmonary bypass. ${ }^{5}$ Although HIT is characterized by thrombocytopenia, bleeding complications are rare; rather, HIT results in a paradoxical prothrombotic disorder with an incidence of thrombosis ranging from $50 \%$ to $89 \%$ in untreated patients. ${ }^{9,14,15}$ Patients can present with acute myocardial infarction, stroke, mesenteric ischemia, ischemic limb necrosis, DVT, and pulmonary embolism. Venous thromboses predominate over arterial thromboses in nonsurgical patients; however, both occur with similar frequency following vascular and cardiac surgery. ${ }^{10,16}$ Five of six patients in our study were readmitted with thrombotic complications and were started on heparin with a subsequent rapid decline in platelet count before being switched to a heparin alternative. We also found that $33.3 \%$ of patients who were readmitted for venous thromboembolic complications were found to be HIT positive during their readmission. This suggests that although recent surgery is in of itself a risk factor VTE, the presence of HITT should be suspected in patients who are readmitted with thrombotic complications after cardiac surgery, even in the absence of significant thrombocytopenia. It has been shown that heparin-dependent antibodies can be detected before the initiation of heparin in patients who go on to develop rapid-onset thrombocytopenia after repeat exposure. ${ }^{7}$ The use of heparin alternatives should therefore be considered in patients readmitted with thrombotic complications after cardiac surgery until they are ruled out for HIT. Depending on patient-specific factors such as renal and liver function, physician experience, and the clinical acuity of thromboembolic disease, direct thrombin and factor $\mathrm{X}$ inhibitors, direct oral anticoagulants, and fondaparinux may be considered in such patients. ${ }^{17,18}$

Interestingly, recent studies have examined the financial burden and resource use associated with HIT in patients undergoing cardiac surgery. Using the National Inpatient Sample database, Seigerman and colleaagues ${ }^{1}$ observed that patients with HIT remained in the hospital twice as long and were nearly twice as likely to be discharged to a skilled nursing facility. Similarly, Aguayo and colleagues ${ }^{19}$ demonstrated that the presence of HIT was associated with longer median duration of stay and increased hospitalization costs. Early recognition and management of HIT is essential for favorable outcomes. The potential suspicion and reduction of new-onset HIT during readmission following cardiac surgery, thus, has significant financial implications as well.

The main limitations of our study are inherent to its retrospective nature and single-center design. It is possible that true incidence of HIT on readmission is greater than we have reported, given that patients with clinically silent thrombotic complications may have gone undetected. Further, there may be patients who developed delayed postoperative HIT but not severe enough to require readmission; these patients also would not be detected. However, given our large sample size and that our incidence rate of HIT on readmission is similar to those reported on index admission, we believe this limitation to be minor. Lastly, as not all patients underwent HIT testing during the index admission, it is possible that the diagnosis of HIT was initially missed. This is unlikely, however, as most patients recovered their platelet counts postoperatively with continued exposure to heparin-based VTE prophylaxis.

\section{CONCLUSIONS}

A significant percentage of patients readmitted with thrombotic complications after cardiac surgery are at risk for having heparin-induced thrombosis, even in the absence of significant thrombocytopenia. The use of non-heparin anticoagulation for the treatment of thrombotic complications in these patients is recommended until HIT status is determined.

\section{Conflict of Interest Statement}

The authors reported no conflicts of interest.

The Journal policy requires editors and reviewers to disclose conflicts of interest and to decline handling or reviewing manuscripts for which they may have a conflict of interest. The editors and reviewers of this article have no conflicts of interest.

\section{References}

1. Seigerman M, Cavallaro P, Itagaki S, Chung I, Chikwe J. Incidence and outcomes of heparin-induced thrombocytopenia in patients undergoing cardiac surgery in North America: an analysis of the nationwide inpatient sample. $J$ Cardiothorac Vasc Anesth. 2014;28:98-102.

2. Solanki J, Shenoy S, Downs E, Palkimas S, Goldman S, Sharma AM. Heparininduced thrombocytopenia and cardiac surgery. Semin Thorac Cardiovasc Surg. 2019;31:335-44.

3. Greinacher A, Eichler P, Lubenow N, Kwasny H, Luz M. Heparin-induced thrombocytopenia with thromboembolic complications: meta-analysis of 2 prospective trials to assess the value of parenteral treatment with lepirudin and its therapeutic aptt range. Blood. 2000;96:846-51.

4. Nazi I, Arnold DM, Moore JC, Smith JW, Ivetic N, Horsewood P, et al. Pitfalls in the diagnosis of heparin-induced thrombocytopenia: a 6-year experience from a reference laboratory. Am J Hematol. 2015;90:629-33.

5. Matthai WH Jr, Cines DB. Towards a diagnosis of heparin-induced thrombocytopenia after cardiopulmonary bypass. J Thromb Haemost. 2004;2:1879-81.

6. Minet V, Dogne JM, Mullier F. Functional assays in the diagnosis of heparininduced thrombocytopenia: a review. Molecules. 2017;22:617.

7. Warkentin TE, Kelton JG. Temporal aspects of heparin-induced thrombocytopenia. N Engl J Med. 2001;344:1286-92.

8. Pouplard C, May MA, Regina S, Marchand M, Fusciardi J, Gruel Y. Changes in platelet count after cardiac surgery can effectively predict the development of pathogenic heparin-dependent antibodies. Br J Haematol. 2005;128:837-41.

9. Warkentin TE, Greinacher A. Heparin-induced thrombocytopenia and cardiac surgery. Ann Thorac Surg. 2003;76:2121-31

10. Shantsila E, Lip GYH, Chong BH. Heparin-induced thrombocytopenia. A contemporary clinical approach to diagnosis and management. Chest. 2009 135:1651-64.

11. Tibayan FA, Leung LL, Burdon TA, Fann JI. Heparin-induced thrombosis without thrombocytopenia. J Thorac Cardiovasc Surg. 2010;139:e6-7.

12. Bigdeli AK, Schmitz C, Brugger D. Heparin-induced thrombosis without thrombocytopenia causing fulminant pulmonary embolism after off-pump coronary artery bypass grafting. Heart Surg Forum. 2009;12:E368-70. 
13. Busche MN, Peters T, Knobloch K, Vogt PM, Rennekampff HO. Heparininduced thrombocytopenia in a nonthrombocytopenic patient with toxic epidermal necrolysis causing fatal outcome: is HIT still a HIT? J Burn Care Res. 2009;30:747-51.

14. Lee GM, Arepally GM. Heparin-induced thrombocytopenia. Hematology Am Soc Hematol Educ Program. 2013;2013:668-74.

15. Warkentin TE, Levine MN, Hirsh J, Horsewood P, Roberts RS, Gent M, et al. Heparin-induced thrombocytopenia in patients treated with lowmolecular-weight heparin or unfractionated heparin. N Engl J Med. 1995; 332:1330-5.

16. Arepally GM, Ortel TL. Heparin-induced thrombocytopenia. Annu Rev Med. 2010;61:77-90.

17. Cuker A, Arepally GM, Chong BH, Cines DB, Greinacher A, Gruel Y, et al. American Society of Hematology 2018 guidelines for management of venous thromboembolism: heparin-induced thrombocytopenia. Blood Adv. 2018;2: 3360-92.

18. Schindewolf M, Steindl J, Beyer-Westendorf J, Schellong S, Dohmen PM, Brachmann J, et al. Use of fondaparinux off-label or approved anticoagulants for management of heparin-induced thrombocytopenia. J Am Coll Cardiol. 2017;70:2636-48.

19. Aguayo E, Sanaiha Y, Seo YJ, Mardock A, Bailey K, Dobaria V, et al. Heparininduced thrombocytopenia in cardiac surgery: incidence, costs, and duration of stay. Surgery. 2018;164:1377-81.

Key Words: HIT, cardiac surgery, thrombotic complications 